\title{
HYPERFINITE DIMENSIONAL REPRESENTATIONS OF SPACES AND ALGEBRAS OF MEASURES
}

\author{
Miloš Ziman AND Pavol Zlatoš
}

\begin{abstract}
Let $\mathbf{X}$ be a locally compact topological space and $\left(X, E, X_{\omega}\right)$ be any triple consisting of a hyperfinite set $X$ in a sufficiently saturated nonstandard universe, a monadic equivalence relation $E$ on $X$, and an $E$-closed galactic set $X_{\omega} \subseteq X$, such that all internal subsets of $X_{\omega}$ are relatively compact in the induced topology and $\mathbf{X}$ is homeomorphic to the quotient $X_{\omega} / E$. We will show that each regular complex Borel measure on $\mathbf{X}$ can be obtained by pushing down the Loeb measure induced by some internal function $X \rightarrow{ }^{*} \mathbb{C}$. The construction gives rise to an isometric isomorphism of the Banach space $\mathbf{M}(\mathbf{X})$ of all regular complex Borel measures on $\mathbf{X}$, normed by total variation, and the quotient $\mathcal{M}_{\omega}(X) / \mathcal{M}_{0}(X)$, for certain external subspaces $\mathcal{M}_{0}(X), \mathcal{M}_{\omega}(X)$ of the hyperfinite dimensional Banach space $* \mathbb{C}^{X}$, with the norm $\|f\|_{1}=\sum_{x \in X}|f(x)|$. If additionally $X=G$ is a hyperfinite group, $X_{\omega}=G_{\omega}$ is a galactic subgroup of $G, E$ is the equivalence corresponding to a normal monadic subgroup $G_{0}$ of $G_{\omega}$, and $\mathbf{G}$ is isomorphic to the locally compact group $G_{\omega} / G_{0}$, then the above Banach space isomorphism preserves the convolution, as well, i.e., $\mathbf{M}(\mathbf{G})$ and $\mathcal{M}_{\omega}(G) / \mathcal{M}_{0}(G)$ are isometrically isomorphic as Banach algebras.
\end{abstract}

\section{Introduction}

A great deal of methods of nonstandard analysis is based on embedding classical mathematical structures into somehow related hyperfinite ones. As a rule, the hyperfinite set (topological space, measure space, etc.) or hyperfinite dimensional vector space $X$, extending the classical object $\mathbf{X}$, is subject to the inclusions $\mathbf{X} \subseteq$ $X \subseteq{ }^{*} \mathbf{X}$, i.e., it is singled out from the nonstandard extension ${ }^{*} \mathbf{X}$ of $\mathbf{X}$. This has the additional advantage of $X$ naturally inheriting the structure from $\mathbf{X}$, via the extension ${ }^{*} \mathbf{X}$, and applicability of the transfer principle.

The method, however, may fail to work that way in presence of some already a little bit more complex algebraic structure on $\mathbf{X}$. For instance, given a group $\mathbf{G}$, there need not be any hyperfinite group $G$ subject to $\mathbf{G} \leq G \leq{ }^{*} \mathbf{G}$. On the other hand, some hyperfinite group $G$ extending $\mathbf{G}$ all the same may (though still need not) exist. And similarly for associative linear algebras over some field. The situation becomes even more complicated for topological groups and Banach algebras. On the other hand, especially for the sake of applications of nonstandard methods to the study of spaces and algebras of functions or measures over $\mathbf{G}$, it is desirable to have $\mathbf{G}$ embedded into some hyperfinite group $G$ and relate somehow the just

1991 Mathematics Subject Classification. Primary 28E05, 43A10; Secondary 03H05, 22D15, 46S20, 54J05.

Key words and phrases. Nonstandard analysis, hyperfinite, Loeb measure, regular complex Borel measure, locally compact group, algebra of measures.

Research supported by a grant by VEGA - Scientific Grant Agency of Slovak Republic

Typeset by $\mathcal{A}_{\mathcal{M S}}$-TEX 
mentioned spaces and algebras to the hyperfinite dimensional space (algebra) $* \mathbb{C}^{G}$. The development led to revival of investigations of local embeddability of groups and algebras into finite groups and finite dimensional algebras and - by involving methods of nonstandard analysis - gave a new, fresh impetus the theory of local approximations of topological groups by finite groups and of Banach algebras by finite dimensional algebras. The present state of the topic is reflected in the monograph [8] by Gordon and in the papers [2], [7], [9], [10], [11].

Our paper contributes namely to this issue. Accordingly, nonstandard mathematical structures become primary objects of our study, considered prior to the classical ones, which, as a rule, are obtained as quotients of certain their substructures. Originally we expected to prove that the Banach algebra $\mathbf{M}(\mathbf{G})$ of regular Borel measures on a locally compact group $\mathbf{G}$, locally approximable by finite groups, is itself locally approximable by finite dimensional algebras, by embedding $\mathbf{M}(\mathbf{G})$ into the nonstandard hull of a hyperfinite dimensional Banach algebra. However, we succeeded to embed it just into a certain explicitly described quotient of such a nonstandard hull. This does not necessarily witness the failure of our goal; equally well it can indicate the need to extend the definition of local approximability.

The plan of the paper is as follows. In section 1 we introduce the notion of $I M G$ space, which is a triple $\left(X, E, X_{\omega}\right)$ consisting of an internal set $X$, a monadic equivalence relation $E$ on $X$ and a galactic $E$-closed set $X_{\omega} \subseteq X$. IMG spaces serve as convenient framework for dealing with nonstandard counterparts of (sufficiently regular) topological spaces. In particular, every Hausdorff locally compact space $\mathbf{X}$ is homeomorphic to the observable trace $X^{b}=X_{\omega} / E$ of some hyperfinite condensating IMG space $\left(X, E, X_{\omega}\right)$. This part is formulated in the spirit (though not in the language) of Vopěnka's alternative set theory, as presented in [29], [30], [12], [31]. However, from technical point of view, the presented results already belong to nonstandard folklore, enabling to omit almost completely the proofs.

In section 2 some results on finite non-negative Loeb measures are modified to Loeb measures induced by complex internal finitely additive measures. Section 3 deals with pushing down complex Loeb measures induced by internal functions $f: X \rightarrow^{*} \mathbb{C}$ on some hyperfinite set $X$. However, instead of focussing just on a single measure, we emphasize the global aspect by analyzing the relations between the internal Banach space $* \mathbb{C}^{X}$, with the norm $\|f\|_{1}=\sum_{x \in X}|f(x)|$, and the classical Banach space $\mathbf{M}(\mathbf{X})$ of regular complex Borel measures on the locally compact space $\mathbf{X}$ represented as the observable trace of some condensating IMG space with base set $X$, normed by the total variation. This line is followed in the subsequent sections 4 and 5, where we show that every measure $\boldsymbol{\mu} \in \mathbf{M}(\mathbf{X})$ can be obtained in this way, and establish the isometric isomorphism $\mathbf{M}(\mathbf{X}) \cong \mathcal{M}_{\omega}(X) / \mathcal{M}_{0}(X)$ for certain external closed $\mathbb{F}^{*} \mathbb{C}$-linear subspaces $\mathcal{M}_{0}(X) \subseteq \mathcal{M}_{\omega}(X) \subseteq{ }^{*} \mathbb{C}^{X}$.

In the last section 6 the notion of $I M G$ group as a nonstandard counterpart of classical topological groups is introduced. For a locally compact group $\mathbf{G}$ representable as the observable trace $\mathbf{G} \cong G^{b}=G_{\omega} / G_{0}$ of some condensating IMG group $\left(G, G_{0}, G_{\omega}\right)$ with hyperfinite $G$ the relation between the internal convolution on the space ${ }^{*} \mathbb{C}^{G}$ and the convolution of measures in $\mathbf{M}(\mathbf{G})$ is investigated. Our final result states that $\mathbf{M}(\mathbf{G})$ and $\mathcal{M}_{\omega}(G) / \mathcal{M}_{0}(G)$ are in fact isometrically isomorphic as Banach algebras.

The reader is assumed to be acquainted with basic as well as with some more involved notions, methods and results of nonstandard analysis, including some usual 
conventions. In particular, some knowledge of the nonstandard approach to topology, of the Loeb measure construction and of internal Banach spaces and their nonstandard hulls is indispensable. Besides the original Robinson's book [25], the standard general reference are the monographs [1] and [28]. For Loeb measure also the original papers by Loeb [18-21], as well as [4], [5], [13], [14] and the survey [6] by Cutland can be consulted. Some recent developments are reflected in [17], [3] and the survey [26] by Ross. The canonic reference for nonstandard Banach space theory is the paper [15] by Henson and Moore.

Our exposition takes place in a nonstandard universe ${ }^{*} \mathbf{V}(\mathbf{I}) \subseteq \mathbf{V}\left({ }^{*} \mathbf{I}\right)$ which is a sufficiently saturated elementary extension of the superstructure $\mathbf{V}(\mathbf{I})$ over some set of individuals I containing at least all (classical) complex numbers. More precisely, we assume that our nonstandard universe is either $\kappa$-saturated for some uncountable cardinal $\kappa$ or even polysaturated, i.e., $\kappa$-saturated for some $\kappa$ bigger than the cardinality of any set in $\mathbf{V}(\mathbf{I})$. However, for the sake of generality, we do not specify the saturation degree $\kappa$ explicitly. Instead we use the term set or system of admissible size referring to (external) sets $\mathcal{S} \subseteq{ }^{*} \mathbf{V}(\mathbf{I})$ of (external) cardinality less than $\kappa$. In the most frequently occurring case of an $\aleph_{1}$-saturated universe (i.e., for $\kappa=\aleph_{1}$ ), a system of admissible size is simply a countable one.

If $\mathcal{A}$ is an internal (Boolean) algebra of subsets of some internal set $X$ then the $\mathcal{A}$-monadic and $\mathcal{A}$-galactic sets are exactly the intersections or unions, respectively, of systems $\mathcal{S} \subseteq \mathcal{A}$ of admissible size. Monadic and galactic sets are just intersections or unions, respectively, of admissibly many internal sets.

Otherwise, we use rather standard set-theoretical notation and terminology. However, the following two remarks seem to be in order.

First, given a set $X$, a relation $R \subseteq X \times X=X^{2}$ and a subset $A \subseteq X$ we denote by $R A=\{x \in X ;(\exists a \in A)(a R x)\}$ the $R$-image of $A$. $A$ is called $R$-closed if $R A \subseteq A$.

Second, if $K \subseteq{ }^{*} \mathbb{C}$ is any (maybe external) subring of the field ${ }^{*} \mathbb{C}$ and $W$ is an internal vector space over ${ }^{*} \mathbb{C}$, then a subset of $W$ is called a $K$-linear subspace of $W$ if it is a subgroup of the additive group of $W$ closed with respect to multiples by scalars from $K$. If $K$ contains finite hypercomplex numbers only, $U$ is a $K$-linear subspace of $W$ and $\mathbf{Z}$ is some vector space over $\mathbb{C}$, then a $K$-linear mapping $\phi: U \rightarrow \mathbf{Z}$ is a homomorphism of their additive groups such that $\phi(a x)={ }^{\circ} a \phi(x)$ for any $a \in K$, $x \in U$. If $W$ is an internal (associative) linear algebra over ${ }^{*} \mathbb{C}$, then a $K$-linear subalgebra of $W$ is a subring of $W$ which is also a $K$-linear subspace of $W$. If $A \subseteq W$ is a $K$-linear subalgebra of $W$ then a $K$-ideal of $A$ is simply an ideal of the ring $A$ which is also a $K$-linear subspace of $W$.

\section{Nonstandard representation of locally compact spaces}

Let a $X$ be a nonempty internal set, $E$ be a monadic equivalence relation on $X$ and $X_{\omega}$ be an E-closed galactic subset of $X$. Then the triple $\left(X, E, X_{\omega}\right)$ is called an $I M G$ space. Intuitively, $E$ is viewed as the relation of infinitesimal nearness or indiscernibility on $X$, and so is $X_{\omega}$ as the set of elements of $X$ lying in front of some horizon - depending on situation, this can be the set of all near-standard or finite (bounded) elements of $X$, or even something else. The former fact is stressed by the notation $x \approx y$ used instead of $(x, y) \in E$ for $x, y \in X$. An IMG space $\left(X, E, X_{\omega}\right)$ is called hyperfinite if $X$ is hyperfinite.

It is an easy exercise in saturation to show that in any IMG space $\left(X, E, X_{\omega}\right)$ 
there is a downward directed system $\mathcal{R}$ of reflexive and symmetric internal relations on $X$, and an upward directed system $\mathcal{V}$ of internal subsets of $X$, both of admissible size, satisfying the following conditions:

$$
\begin{aligned}
& E=\bigcap \mathcal{R}, \quad \text { and } \quad(\forall R \in \mathcal{R})(\exists S \in \mathcal{R})(S \circ S \subseteq R) \text {, } \\
& X_{\omega}=\bigcup \mathcal{V}, \quad \text { and } \quad(\forall U \in \mathcal{V})(\exists V \in \mathcal{V})(\exists R \in \mathcal{R})(R U \subseteq V) \text {. }
\end{aligned}
$$

Then $\mathcal{R}$ becomes a base of a uniformity on $X$ (non-Hausdorff, unless $E=\operatorname{Id}_{X}$ ). A set $Y \subseteq X$ is open in the induced topology if and only if for each $y \in Y$ there is an internal set $A$ such that $E\{y\} \subseteq A \subseteq Y$. In particular, $X_{\omega}$ is an open subset of $X$. The closure of any set $Y \subseteq X$ is $\bigcap_{R \in \mathcal{R}} R Y$; for internal $Y$ this is equal to $\left(\bigcap_{R \in \mathcal{R}} R\right) Y=E Y$.

Let $x \mapsto x^{b}$ denote the restriction of the canonic projection $X \rightarrow X / E$ to $X_{\omega}$. In some sense, it is analogous to the standard part map. For each set $Y \subseteq X$ we have

$$
Y^{b}=\left\{y^{b} ; y \in Y \cap X_{\omega}\right\}
$$

in particular,

$$
X^{b}=X_{\omega}^{b}=X_{\omega} / E
$$

becomes a Hausdorff completely regular space, with topology induced by the uniformity with base formed by the relations

$$
R^{b}=\left\{\left(x^{b}, y^{b}\right) ;(x, y) \in R \cap X_{\omega}^{2}\right\}
$$

where $R \in \mathcal{R}$. As a uniform space, $X^{b}$ is complete. Conversely, for any set $\mathbf{Y} \subseteq X^{b}$ we denote by

$$
\mathbf{Y}^{\sharp}=\left\{y \in X_{\omega} ; y^{b} \in \mathbf{Y}\right\}
$$

the preimage of $\mathbf{Y}$ under the mapping $x \mapsto x^{b}$.

The topological space $X^{b}$ could be called the nonstandard hull of the IMG space $\left(X, E, X_{\omega}\right)$; however, we prefer to call it the observable trace of $\left(X, E, X_{\omega}\right)$, and reserve the term "nonstandard hull" for a more specific situation when $X={ }^{*} \mathbf{X}$ is the nonstandard extension of some classical topological space $\mathbf{X}$. Similarly, the mapping $x \mapsto x^{b}$ is called the observable trace map, and $x^{b}$ and $Y^{b}$ are referred to as the observable trace of $x \in X_{\omega}$ and of $Y \subseteq X$, respectively. The set $\mathbf{Y}^{\sharp}$ is called the pretrace of the set $\mathbf{Y} \subseteq X^{b}$.

A system $\mathcal{B}$ of internal subsets of $X$ is called a base of the IMG space $\left(X, E, X_{\omega}\right)$ if it is closed under complements and finite unions (i.e., it is a subalgebra of the algebra ${ }^{*} \mathcal{P}(X)$ of all internal subsets of $\left.X\right)$, and for any $x \in X_{\omega}$ and any internal set $A \subseteq X$, satisfying $E\{x\} \subseteq A$, there is a $B \in \mathcal{B}$ such that $E\{x\} \subseteq B \subseteq A$. If $\mathcal{B}$ is a base of $\left(X, E, X_{\omega}\right)$ then the system

$$
\mathcal{B}^{b}=\left\{B^{b} ; B \in \mathcal{B}\right\}
$$

is a fairly good substitute of a base of the topology in $X^{b}$ : namely, for any $x \in X_{\omega}$ the system of all sets $B^{b}$, such that $E\{x\} \subseteq B \in \mathcal{B}$, is a neighborhood base of the point $x^{b} \in X^{b}$ (consisting of closed sets). 
Following [22], [23], an IMG space $\left(X, E, X_{\omega}\right)$ is called condensating if any infinite internal set $A \subseteq X_{\omega}$ contains at least two distinct elements $x \approx y$. This is equivalent to relative compactness of all internal subsets of $X_{\omega}$. As a consequence, the observable trace $X^{b}$ is locally compact. Moreover, the compact subsets of $X_{\omega}$ are exactly those of the form $E A$ for some internal subset $A \subseteq X_{\omega}$. Similarly, the compact subsets of $X^{b}$ are exactly their observable traces, i.e., sets of the form $(E A)^{b}=A^{b}$ for internal sets $A \subseteq X_{\omega}$.

If all the sets in $\mathcal{V}$ are relatively compact, then, for each relation $R \in \mathcal{R}$ and each set $V \in \mathcal{V}$, there is a finite set $F$ such that $F \subseteq V \subseteq R F$. Thus the systems $\mathcal{R}, \mathcal{V}$ can be supplemented by a system of finite sets $\left(F_{R, V}\right)$, where $R \in \mathcal{R}, V \in \mathcal{V}$, satisfying

$$
F_{R, V} \subseteq V \subseteq R F_{R, V}, \quad \text { and } \quad\left(\forall x, y \in F_{R, V}\right)(x R y \Rightarrow x=y)
$$

Then the set

$$
D=\bigcup_{\substack{R \in \mathcal{R} \\ V \in \mathcal{V}}} F_{R, V},
$$

as union of admissibly many finite sets, is of admissible size, as well. Obviously, $D$ is dense in $X_{\omega}$ and so is $D^{b}$ in $X^{b}$. Moreover, $x \not z y$ for any distinct $x, y \in D$.

Let $\mathcal{B}$ be the subalgebra of ${ }^{*} \mathcal{P}(X)$ generated by all the sets of the form $R\{d\}$, where $R \in \mathcal{R}, d \in D$. It can easily be seen that $\mathcal{B}$ is of admissible size and forms a base of the condensating IMG space $\left(X, E, X_{\omega}\right)$.

1.1. Proposition. (a) Let $\mathbf{X}$ be a Hausdorff completely regular topological space. Then $\mathbf{X}$ is homeomorphic to a subspace of the observable trace $X^{b}=X_{\omega} / E$ of some IMG space $\left(X, E, X_{\omega}\right)$.

(b) Let $\mathbf{X}$ be a Hausdorff locally compact topological space. Then $\mathbf{X}$ is homeomorphic to the observable trace $X^{b}=X_{\omega} / E$ of some condensating IMG space $\left(X, E, X_{\omega}\right)$.

Moreover, both in (a) and (b), one can additionally assume $X$ to be hyperfinite.

Proof. (a) Let $X$ be any internal set such that $\mathbf{X} \subseteq X \subseteq{ }^{*} \mathbf{X}$, hyperfinite if desirable. As $\mathbf{X}$ is completely regular, its topology is induced by some uniformity $\mathcal{U}$. Put $E=\bigcap_{\mathbf{U} \in \mathcal{U}}{ }^{*} \mathbf{U} \cap X^{2}$. Let $\mathcal{S}$ be any open cover of $\mathbf{X}$ and $X_{\omega}=\bigcup_{\mathbf{S} \in \mathcal{S}}{ }^{*} \mathbf{S} \cap X$. Once our nonstandard universe is at least $\kappa$-saturated, where $\kappa$ is any uncountable cardinal bigger both than $|\mathcal{S}|$ and the minimal cardinality of a base of $\mathcal{U}$, then the triple $\left(X, E, X_{\omega}\right)$ is an IMG space with all the needed properties.

(b) Let $X$ and $E$ be as in (a), and $\mathcal{S}$ be any cover of $\mathbf{X}$ by relatively compact open sets. Then $X_{\omega}=\operatorname{ns}\left({ }^{*} \mathbf{X}\right)$ and $\mathbf{X} \cong X^{b}$.

One should be aware, however, that the particular form of the IMG space $\left(X, E, X_{\omega}\right)$ from the proof is not obligatory. One can equally well obtain $\mathbf{X}$ as (a subspace of) the observable trace of an IMG space given in advance, prior to and independently of the nonstandard extension ${ }^{*} \mathbf{X}$.

In particular, (b) applies to the space $\mathbb{C}$ of all complex numbers, which can be represented as the quotient $\mathbb{F}^{*} \mathbb{C} / \mathbb{I}^{*} \mathbb{C}$, arising from the condensating IMG space $\left(* \mathbb{C}, \approx, \mathbb{F}^{*} \mathbb{C}\right)$, where $\approx$ denotes the relation of infinitesimal nearness on ${ }^{*} \mathbb{C}$, and $\mathbb{I}^{*} \mathbb{C}$ and $\mathbb{F}^{*} \mathbb{C}$ denote the sets of all infinitesimal and all finite hypercomplex numbers, respectively. 
Similarly, the one point compactification of the (locally compact) observable trace $X^{b}$ of a condensating IMG space $\left(X, E, X_{\omega}\right)$ can be represented as the quotient $X / E^{\prime}$ of $X$ with respect to the monadic equivalence relation

$$
E^{\prime}=E \cup\left(X \backslash X_{\omega}\right)^{2},
$$

i.e., as the observable trace of the condensating IMG space $\left(X, E^{\prime}, X\right)$ with compact internal galaxy $X_{\omega}^{\prime}=X$.

Every internal $S$-continuous function $f: X \rightarrow{ }^{*} \mathbb{C}$ with finite internal supremum norm

$$
\|f\|_{\infty}={ }^{*} \sup _{x \in X}|f(x)|
$$

defines a bounded uniformly continuous function, $f^{b}: X^{b} \rightarrow \mathbb{C}$, called the observable trace of $f$, where

$$
f^{b}\left(x^{b}\right)={ }^{\circ} f(x),
$$

for $x \in X_{\omega}$. The other way round, every bounded continuous function $\mathbf{f}: X^{b} \rightarrow \mathbb{C}$, vanishing at infinity, is of the form $\mathbf{f}=f^{b}$ for some internal $S$-continuous function $f: X \rightarrow{ }^{*} \mathbb{C}$, satisfying $\|f\|_{\infty}<\infty$ and $f(x) \approx 0$ for each $x \in X \backslash X_{\omega}$. Indeed, f can be extended to a unique continuous function $X / E^{\prime} \rightarrow \mathbb{C}$. Then the existence of $f$ follows from the compactness of $X / E^{\prime}$ by the virtue of saturation.

This observation gives rise to a nonstandard representation of the classical Banach space $\mathbf{C}_{0}\left(X^{b}\right)$ of all continuous functions $\mathbf{f}: X^{b} \rightarrow \mathbb{C}$ vanishing at infinity. Let us denote

$$
\begin{aligned}
\mathbb{I}_{\infty}{ }^{*} \mathbb{C}^{X} & =\left\{f \in{ }^{*} \mathbb{C}^{X} ;\|f\|_{\infty} \approx 0\right\}, \\
\mathbb{F}_{\infty}{ }^{*} \mathbb{C}^{X} & =\left\{f \in{ }^{*} \mathbb{C}^{X} ;\|f\|_{\infty}<\infty\right\}, \\
\mathcal{C}(X) & =\left\{f \in \mathbb{F}_{\infty}{ }^{*} \mathbb{C}^{X} ; f \text { is } S \text {-continuous }\right\}, \\
\mathcal{C}_{\omega}(X) & =\left\{f \in \mathcal{C}(X) ;\left(\forall x \in X \backslash X_{\omega}\right)(f(x) \approx 0)\right\} .
\end{aligned}
$$

Then there is a chain of $\mathbb{F}^{*} \mathbb{C}$-linear subspaces of the internal vector space ${ }^{*} \mathbb{C}^{X}$ :

$$
\mathbb{I}_{\infty}^{*} \mathbb{C}^{X} \subseteq \mathcal{C}_{\omega}(X) \subseteq \mathcal{C}(X) \subseteq \mathbb{F}_{\infty}{ }^{*} \mathbb{C}^{X}
$$

and the quotient $\mathcal{C}_{\omega}(X) / \mathbb{I}_{\infty}{ }^{*} \mathbb{C}^{X}$ becomes a (classical) Banach space, isometrically isomorphic to $\mathbf{C}_{0}\left(X^{b}\right)$. This gives the representation of

$$
\mathbf{C}_{0}\left(X^{b}\right) \cong \mathcal{C}_{\omega}(X) / \mathbb{I}_{\infty}^{*} \mathbb{C}^{X}
$$

as a closed subspace of the observable trace (nonstandard hull) $\mathbb{F}_{\infty}{ }^{*} \mathbb{C}^{X} / \mathbb{I}_{\infty}{ }^{*} \mathbb{C}^{X}$ of the internal Banach space $\left({ }^{*} \mathbb{C}^{X},\|\cdot\|_{\infty}\right)$. If desirable, one can take a hyperfinite $X$, yielding a hyperfinite dimensional $* \mathbb{C}^{X}$.

\section{Complex Loeb measures}

Let $X$ be an internal set, $\mathcal{A}$ be an internal algebra of (internal) subsets of $X$ and $\nu: \mathcal{A} \rightarrow{ }^{*} \mathbb{R}$ be an internal finitely additive (hence hyperfinitely additive) nonnegative measure. We additionally assume that $\nu$ is finite, i.e., $\nu(A)$ is a finite hyperreal number for each $A \in \mathcal{A}$, in symbols, $\nu(X)<\infty$. Then the assignment

$$
{ }^{\circ} \nu(A)={ }^{\circ}(\nu(A))
$$


defines a finite, finitely additive non-negative measure ${ }^{\circ} \nu: \mathcal{A} \rightarrow \mathbb{R}$. According to the results of Landers and Rogge [17, theorem 1], it has a unique extension to a finite $\sigma$-additive non-negative measure $L(\nu): \widetilde{\mathcal{A}} \rightarrow \mathbb{R}$, defined on the $\sigma$-algebra $\widetilde{\mathcal{A}}$ generated by the system of all $\mathcal{A}$-monadic sets (if $\kappa=\aleph_{1}$ then $\widetilde{\mathcal{A}}$ is simply the $\sigma$-algebra generated by $\mathcal{A})$. Then $\lambda=L(\nu)$ is called the Loeb measure induced by the internal measure $\nu$. It can be explicitly described as follows: If $B \in \widetilde{\mathcal{A}}$, then

$$
\lambda(B)=\inf \left\{{ }^{\circ} \nu(A) ; A \in \mathcal{A} \& B \subseteq A\right\}=\sup \left\{{ }^{\circ} \nu(A) ; A \in \mathcal{A} \& A \subseteq B\right\},
$$

and $\lambda(A \triangle B)=0$ for some $A \in \mathcal{A}$. Moreover, the Loeb measure is partially $\tau$-smooth in the following sense: For any directed system of sets $\mathcal{S} \subseteq \mathcal{A}$ of admissible size we have either

$$
\lambda(\bigcap \mathcal{S})=\inf \{\lambda(S) ; S \in \mathcal{S}\}, \quad \text { or } \quad \lambda(\bigcup \mathcal{S})=\sup \{\lambda(S) ; S \in \mathcal{S}\},
$$

depending on whether $\mathcal{S}$ is downward or upward directed.

Remark. (a) Sometimes by Loeb measure either the extension of ${ }^{\circ} \nu$ just to the $\sigma$-algebra $\sigma \mathcal{A}$ generated by $\mathcal{A}$, or yet its Carathéodory completion, i.e., its extension to the $\sigma$-algebra $L(\mathcal{A}, \nu)$ of all Loeb measurable sets is meant. In general we have $\sigma \mathcal{A} \subseteq \widetilde{\mathcal{A}} \subseteq L(\mathcal{A}, \nu)$. However, unless the nonstandard universe is only $\aleph_{1}$-saturated, $\sigma \mathcal{A}$ does not necessarily contain all the $\mathcal{A}$-monadic and $\mathcal{A}$-galactic sets. The algebra $\widetilde{\mathcal{A}}$, besides containing them, has the advantage that its members are measurable with respect to all Loeb measures induced by finite internal finitely additive non-negative measures $\nu: \mathcal{A} \rightarrow{ }^{*} \mathbb{R}$.

(b) In [17] universal Loeb mesurability of sets in $\widetilde{\mathcal{A}}$ and partial $\tau$-smoothness of Loeb measures just for finite internal finitely additive measures $\nu$ on algebras of the form $\mathcal{A}={ }^{*} \mathcal{A}_{0}$ was proved. However, their proof works for any internal algebra $\mathcal{A}$ almost without any change.

If $\nu: \mathcal{A} \rightarrow{ }^{*} \mathbb{C}$ is an internal complex (hyper)finitely additive measure, then its internal variation $|\nu|: \mathcal{A} \rightarrow{ }^{*} \mathbb{R}$ is defined by

$$
|\nu|(A)={ }^{*} \sup _{\beta} \sum_{B \in \beta}|\nu(B)|,
$$

with ${ }^{*} \sup _{\beta}$ denoting the internal supremum over all hyperfinite partitions $\beta \subseteq \mathcal{A}$ of the set $A \in \mathcal{A}$. One can show that it is an internal finitely additive nonnegative measure, again. Assuming that $|\nu|$ is finite, i.e., the total internal variation $\|\nu\|=|\nu|(X)$ of $\nu$ is finite, the original measure $\nu$ can be expressed as the Jordan decomposition $\nu=\left(\nu_{1}-\nu_{2}\right)+\mathrm{i}\left(\nu_{3}-\nu_{4}\right)$ for certain finite internal non-negative finitely additive measures $\nu_{1}, \nu_{2}, \nu_{3}, \nu_{4}: \mathcal{A} \rightarrow{ }^{*} \mathbb{R}$. Denoting by $\lambda_{j}$ the Loeb measure induced by $\nu_{j}$, one can formally put $\lambda=\left(\lambda_{1}-\lambda_{2}\right)+\mathrm{i}\left(\lambda_{3}-\lambda_{4}\right)$, and call $\lambda=L(\nu)$ the Loeb measure induced by the internal complex measure $\nu$, again. Then $\lambda(A)={ }^{\circ} \nu(A)$ keeps holding (and makes sense) for all $A \in \mathcal{A}$.

Using the Jordan decomposition the previous accounts on non-negative Loeb measures can be generalized to the complex framework, and the equality of the Loeb measure $L(|\nu|)$, induced by the internal variation $|\nu|$ of the internal measure $\nu$, and the (external) variation $|\lambda|$ of the Loeb measure $\lambda=L(\nu)$ can be established. Recall that the latter is defined by

$$
|\lambda|(B)=\sup _{\gamma} \sum_{C \in \gamma}|\lambda(C)|
$$


for $B \in \widetilde{\mathcal{A}}$, with $\sup _{\gamma}$ denoting the supremum over all finite partitions $\gamma \subseteq \widetilde{\mathcal{A}}$ of the set $B \in \widetilde{\mathcal{A}}$; then the total variation of $\lambda$ is $\|\lambda\|=|\lambda|(X)$.

2.1. Proposition. Let $X$ be an internal set, $\mathcal{A}$ be an internal algebra of subsets of $X$, and $\nu: \mathcal{A} \rightarrow{ }^{*} \mathbb{C}$ be an internal complex finitely additive measure such that $\|\nu\|<\infty$. Then the Loeb measure $\lambda=L(\nu): \widetilde{\mathcal{A}} \rightarrow \mathbb{C}$ is a $\sigma$-additive complex measure extending ${ }^{\circ} \nu$, for any $B \in \widetilde{\mathcal{A}}$ there is an $A \in \mathcal{A}$ such that $|\lambda|(A \triangle B)=0$ and, for each standard $\varepsilon>0$, the following two conditions hold:

(a) there is an $A^{\prime} \in \mathcal{A}$ such that $A^{\prime} \subseteq B$, and $|\lambda(C)|<\varepsilon$, whenever $C \in \widetilde{\mathcal{A}}$ satisfies $C \subseteq B \backslash A^{\prime}$;

(b) there is an $A^{\prime \prime} \in \mathcal{A}$ such that $B \subseteq A^{\prime \prime}$, and $|\lambda(C)|<\varepsilon$, whenever $C \in \widetilde{\mathcal{A}}$ satisfies $C \subseteq A^{\prime \prime} \backslash B$.

Moreover, the variation $|\lambda|$ of $\lambda$ is the Loeb measure induced by the internal variation $|\nu|$ of $\nu$, i.e.,

$$
|\lambda|=|L(\nu)|=L(|\nu|),
$$

in particular, $\|\lambda\|={ }^{\circ}\|\nu\|<\infty$.

Proof. We will prove just the last statement. To this end it is enough to show

$$
|\lambda|(A)={ }^{\circ}|\nu|(A)
$$

for any $A \in \mathcal{A}$. Let the sets $B_{1}, \ldots, B_{n} \in \widetilde{\mathcal{A}}$ form a finite partition of $A$. Take any standard $\varepsilon>0$. Then there are sets $A_{i} \in \mathcal{A}$ such that $A_{i} \subseteq B_{i}$ and $\left|\lambda\left(B_{i} \backslash A_{i}\right)\right|<\varepsilon / n$. Therefore,

$$
\sum_{i=1}^{n}\left|\lambda\left(B_{i}\right)\right|<\sum_{i=1}^{n}\left|\lambda\left(A_{i}\right)\right|+\varepsilon=\sum_{i=1}^{n}\left|{ }^{\circ} \nu\left(A_{i}\right)\right|+\varepsilon \leq{ }^{\circ}|\nu|(A)+\varepsilon,
$$

since the sets $A_{i}$ are pairwise disjoint. As $\varepsilon$ was arbitrary, we have $|\lambda|(A) \leq{ }^{\circ}|\nu|(A)$.

To prove the converse, take some hyperfinite partition $\left\{A_{1}, \ldots, A_{m}\right\} \subseteq \mathcal{A}$ of $A$, and any standard $\varepsilon>0$. Let $c_{j}=\left|\nu\left(A_{j}\right)\right| / \nu\left(A_{j}\right)$ if $\nu\left(A_{j}\right) \neq 0$, and $c_{j}=1$ otherwise. As $a=|\nu|(A)$ is finite, there is a finite integer $n>2 \pi a / \varepsilon$; put $d_{k}=\exp (2 \pi \mathrm{i} k / n)$, for $0 \leq k<n$. Then the set $\{1, \ldots, m\}$ can be partitioned into $n$ hyperfinite sets $J_{0}, \ldots, J_{n-1}$ such that $\left|c_{j}-d_{k}\right|<\varepsilon / 2 a$ for $j \in J_{k}$. The sets $B_{k}=\bigcup_{j \in J_{k}} A_{j} \in \mathcal{A}$, $0 \leq k<n$, form a finite partition of $A$. Therefore,

$$
\begin{aligned}
\sum_{j=1}^{m}\left|\nu\left(A_{j}\right)\right| & =\sum_{k=0}^{n-1} \sum_{j \in J_{k}} c_{j} \nu\left(A_{j}\right)=\sum_{k=0}^{n-1}\left(d_{k} \nu\left(B_{k}\right)+\sum_{j \in J_{k}}\left(c_{j}-d_{k}\right) \nu\left(A_{j}\right)\right) \\
& <\sum_{k=0}^{n-1}\left|\nu\left(B_{k}\right)\right|+\frac{\varepsilon}{2 a} \sum_{j=1}^{m}\left|\nu\left(A_{j}\right)\right| \leq \sum_{k=0}^{n-1}\left|\nu\left(B_{k}\right)\right|+\frac{\varepsilon}{2} \\
& <\sum_{k=0}^{n-1}\left|\lambda\left(B_{k}\right)\right|+\varepsilon \leq|\lambda|(A)+\varepsilon .
\end{aligned}
$$

This proves ${ }^{\circ}|\nu|(A) \leq|\lambda|(A)$.

The partial $\tau$-smoothness of complex Loeb measures can be formulated in terms of limits of nets over (downward or upward) directed systems of sets. 
2.2. Proposition. Let $X, \mathcal{A}$ and $\nu$ be as above, and $\lambda=L(\nu)$. Then for any directed system $\mathcal{S} \subseteq \mathcal{A}$ of admissible size we have either

$$
\lambda(\bigcap \mathcal{S})=\lim _{S \in \mathcal{S} \downarrow} \lambda(S), \quad \text { or } \quad \lambda(\bigcup \mathcal{S})=\lim _{S \in \mathcal{S} \uparrow} \lambda(S),
$$

depending on whether $\mathcal{S}$ is downward or upward directed.

Partial $\tau$-smoothness of Loeb measures implies the following strengthening of the first part of proposition 2.1 for monadic and galactic sets.

2.3. Proposition. Let $X, \mathcal{A}$ and $\nu$ be as above, and $\lambda=L(\nu)$.

(a) If $B \in \widetilde{\mathcal{A}}$ is an $\mathcal{A}$-monadic set, then there is an $A \in \mathcal{A}$ such that $A \subseteq B$ and $\lambda(B) \approx \nu(A)$.

(b) Dually, if $B \in \widetilde{\mathcal{A}}$ is an $\mathcal{A}$-galactic set, then there is an $A \in \mathcal{A}$ such that $B \subseteq A$ and $\lambda(B) \approx \nu(A)$.

Proof. It suffices to prove just (a), (b) then follows by passing to complements. We can assume that $B \notin \mathcal{A}$ and $B=\bigcap \mathcal{S}$ for some downward directed system $\mathcal{S} \subseteq \mathcal{A}$ of admissible size. By saturation, there is a hyperfinite downward directed system $\mathcal{H} \subseteq \mathcal{A}$ extending $\mathcal{S}$. By proposition 2.2,

$$
\lambda(B)=\lim _{S \in \mathcal{S} \downarrow} \lambda(S)=\lim _{S \in \mathcal{S} \downarrow}{ }^{\circ} \nu(S) .
$$

Thus there is an $H \in \mathcal{H} \backslash \mathcal{S}$ such that $\lambda(B) \approx \nu(A)$ whenever $H \subseteq A \in \mathcal{H} \backslash \mathcal{S}$.

If $X$ is hyperfinite and $\mathcal{A}={ }^{*} \mathcal{P}(X)$ is the algebra of all internal subsets of $X$, then any internal finitely additive measure $\nu:{ }^{*} \mathcal{P}(X) \rightarrow{ }^{*} \mathbb{C}$ is determined by its values on singletons, i.e., by the function $f: X \rightarrow{ }^{*} \mathbb{C}$, given by $f(x)=\nu\{x\}$, for $x \in X$. Conversely, every internal function $f: X \rightarrow{ }^{*} \mathbb{C}$ defines an internal finitely additive measure $\nu_{f}:{ }^{*} \mathcal{P}(X) \rightarrow{ }^{*} \mathbb{C}$ by

$$
\nu_{f}(A)=\sum_{x \in A} f(x)
$$

for $A \in{ }^{*} \mathcal{P}(X)$. Then the Loeb measure induced by the internal measure $\nu_{f}$ will be denoted by $\lambda_{f}$ and referred to as the Loeb measure induced by the internal function $f$. In this case, $\left|\nu_{f}\right|=\nu_{|f|}$, in particular,

$$
\left\|\nu_{f}\right\|=\|f\|_{1}=\sum_{x \in X}|f(x)|
$$

As a consequence, we have the following

2.4. Proposition. Let $X$ be a hyperfinite set and $f: X \rightarrow{ }^{*} \mathbb{C}$ be an internal function such that $\|f\|_{1}<\infty$. Then the Loeb measure $\lambda_{f}$ is a $\sigma$-additive, partially $\tau$ smooth complex measure, defined on the $\sigma$-algebra $\widetilde{*}(X)$ generated by all monadic (and galactic) subsets of $X$, with finite total variation $\left\|\lambda_{f}\right\|={ }^{\circ}\|f\|_{1}$.

The adjustment of the remaining parts of propositions 2.1-3, to this particular situation is left to the reader. 


\section{Pushing down Loeb measures}

Through this and the following two sections $\mathbf{X}$ will be a Hausdorff locally compact space, represented as the observable trace $\mathbf{X}=X_{\omega} / E=X^{b}$ of some hyperfinite condensating IMG space $\left(X, E, X_{\omega}\right)$, with a base $\mathcal{B} \subseteq{ }^{*} \mathcal{P}(X)$ of admissible size.

Let us start with the observation that the set $X_{\omega}=\bigcup \mathcal{V}$ itself belongs to the algebra $\widetilde{* P}(X)$, and so do all the sets $E A=\bigcap_{R \in \mathcal{R}} R A$ for any internal $A \subseteq X$. Thus the system

$$
\widetilde{{ }^{*} \mathcal{P}}\left(X_{\omega}\right)=\widetilde{* \mathcal{P}}(X) \cap \mathcal{P}\left(X_{\omega}\right)=\left\{B \in \widetilde{* \mathcal{P}}(X) ; B \subseteq X_{\omega}\right\}
$$

is the least $\sigma$-algebra of subsets of $X_{\omega}$ containing all its monadic and galactic subsets.

Let $\mathrm{Bo}(\mathbf{X})$ denote the $\sigma$-algebra of all Borel subsets of $\mathbf{X}$.

3.1. Proposition. If $\mathbf{Y} \in \operatorname{Bo}(\mathbf{X})$ then $\mathbf{Y}^{\sharp} \in \widetilde{* P}\left(X_{\omega}\right)$. Consequently,

$$
\operatorname{Bo}(\mathbf{X})^{\sharp}=\left\{\mathbf{Y}^{\sharp} ; \mathbf{Y} \in \operatorname{Bo}(\mathbf{X})\right\}
$$

is a $\sigma$-subalgebra of the $\sigma$-algebra ${ }^{* \mathcal{P}}\left(X_{\omega}\right)$, and the observable trace map $x \mapsto x^{b}$, $\left(X_{\omega}, \widetilde{\mathcal{P}}\left(X_{\omega}\right)\right) \rightarrow(\mathbf{X}, \mathrm{Bo}(\mathbf{X}))$ is surjective and measurable.

Proof. As the pretrace operation $\mathbf{Y} \mapsto \mathbf{Y}^{\sharp}$ preserves the set-theoretical difference and arbitrary unions and intersections, it suffices to show that $\mathbf{Y}^{\sharp} \in \widetilde{{ }^{*} \mathcal{P}}\left(X_{\omega}\right)$ for each open set $\mathbf{Y} \subseteq \mathbf{X}$. Then $\mathbf{Y}^{\sharp}$ is open in $X_{\omega}$, as well, hence it can be written as the union

$$
\mathbf{Y}^{\sharp}=\bigcup\left\{B \in \mathcal{B} ;\left(\exists y \in \mathbf{Y}^{\sharp}\right)\left(E\{y\} \subseteq B \subseteq \mathbf{Y}^{\sharp}\right)\right\} .
$$

Thus $\mathbf{Y}^{\sharp}$ is even a galactic set.

If $f: X \rightarrow{ }^{*} \mathbb{C}$ is an internal function such that $\|f\|_{1}<\infty$, then the Loeb measure $\lambda_{f}$ can be restricted to the $\sigma$-algebra $\widetilde{*}\left(X_{\omega}\right)$, as well as to its $\sigma$-subalgebra $\operatorname{Bo}(\mathbf{X})^{\sharp}$. Thus one can put

$$
\boldsymbol{\theta}_{f}(\mathbf{B})=\lambda_{f}\left(\mathbf{B}^{\sharp}\right),
$$

for $\mathbf{B} \in \operatorname{Bo}(\mathbf{X})$.

3.2. Proposition. Let $f: X \rightarrow{ }^{*} \mathbb{C}$ be an internal function such that $\|f\|_{1}<\infty$. Then $\boldsymbol{\theta}_{f}: \mathrm{Bo}(\mathbf{X}) \rightarrow \mathbb{C}$ is a regular complex Borel measure on $\mathbf{X}$, with total variation $\left\|\boldsymbol{\theta}_{f}\right\| \leq{ }^{\circ}\|f\|_{1}$. If, additionally,

$$
\nu_{f}(A)=\sum_{x \in A} f(x) \approx 0
$$

for any internal set $A \subseteq X \backslash X_{\omega}$, then $\lambda_{f}$ is concentrated on $X_{\omega}$ and

$$
\boldsymbol{\theta}_{f}(\mathbf{X})=\lambda_{f}\left(X_{\omega}\right) \approx \sum_{x \in X} f(x)=\nu_{f}(X)
$$

Proof. Concerning the first part of the theorem, in view of propositions 2.1 and 2.4 it remains to prove just the inner-compact regularity of $\boldsymbol{\theta}_{f}$, i.e.,

$$
\left|\boldsymbol{\theta}_{f}\right|(\mathbf{B})=\sup \left\{\left|\boldsymbol{\theta}_{f}\right|(\mathbf{K}) ; \mathbf{K} \subseteq \mathbf{B} \text { is compact }\right\},
$$


for every Borel set $\mathbf{B} \subseteq \mathbf{X}$. This can be done in an obvious way.

Turning to the second part of the theorem, we have $\boldsymbol{\theta}_{f}(\mathbf{X})=\lambda_{f}\left(X_{\omega}\right)$ by the definition. According to proposition $2.3, \lambda\left(X_{\omega}\right) \approx \nu_{f}(Y)$ for some internal set $Y$ such that $X_{\omega} \subseteq Y \subseteq X$. Then $X \backslash Y \subseteq X \backslash X_{\omega}$, hence $\nu_{f}(X \backslash Y) \approx 0$, and

$$
\nu_{f}(X)=\nu_{f}(Y)+\nu_{f}(X \backslash Y) \approx \lambda_{f}\left(X_{\omega}\right)=\boldsymbol{\theta}_{f}(\mathbf{X}) .
$$

Let us introduce the following notation:

$$
\begin{aligned}
\mathbb{I}_{1}{ }^{*} \mathbb{C}^{X} & =\left\{f \in{ }^{*} \mathbb{C}^{X} ;\|f\|_{1} \approx 0\right\}, \\
\mathbb{F}_{1}{ }^{*} \mathbb{C}^{X} & =\left\{f \in{ }^{*} \mathbb{C}^{X} ;\|f\|_{1}<\infty\right\}, \\
\mathcal{M}_{\omega}(X) & =\left\{f \in \mathbb{F}_{1}{ }^{*} \mathbb{C}^{X} ;\left(\forall^{\text {int }} A \subseteq X \backslash X_{\omega}\right)\left(\left|\nu_{f}\right|(A) \approx 0\right)\right\} .
\end{aligned}
$$

Additionally, $\mathbf{M}(\mathbf{X})$ denotes the classical Banach space of all (inner-compact) regular complex Borel measures on $\mathbf{X}$, with the total variation norm-each $\boldsymbol{\mu} \in \mathbf{M}(\mathbf{X})$ is indeed outer-open regular and satisfies $\|\boldsymbol{\mu}\|=|\boldsymbol{\mu}|(\mathbf{X})<\infty$, see, e.g., [27].

The following two facts are obvious.

3.3. Proposition. (a) $\mathbb{I}_{1} * \mathbb{C}^{X} \subseteq \mathcal{M}_{\omega}(X) \subseteq \mathbb{F}_{1}{ }^{*} \mathbb{C}^{X}$ are $\mathbb{F} * \mathbb{C}$-linear subspaces of the internal Banach space $\left(* \mathbb{C}^{X},\|\cdot\|_{1}\right)$.

(b) $\boldsymbol{\theta}: \mathbb{F}_{1}{ }^{*} \mathbb{C}^{X} \rightarrow \mathbf{M}(\mathbf{X})$ is a bounded $\mathbb{F}^{*} \mathbb{C}$-linear mapping.

From the last part of proposition 3.2 it follows that the condition $f \in \mathcal{M}_{\omega}(X)$ is even stronger than necessary to guarantee $\boldsymbol{\theta}_{f}(\mathbf{X}) \approx \nu_{f}(X)$. On the other hand, as shown by the following, rather trivial example, even this condition is not sufficient to ensure $\left|\boldsymbol{\theta}_{f}\right|(\mathbf{X}) \approx\left|\nu_{f}\right|(X)$ or, which is the same, $\left\|\boldsymbol{\theta}_{f}\right\| \approx\|f\|_{1}$.

3.4. Example. Let $u, v \in X_{\omega}$ be two distinct elements such that $u \approx v$. Let the internal function $f: X \rightarrow{ }^{*} \mathbb{C}$ be defined by $f(u)=1, f(v)=-1$ and $f(x)=0$ for any other $x \in X$. Then $\|f\|_{1}=2$, and for each internal set $A \subseteq X \backslash X_{\omega}$ we even have $\sum_{x \in A}|f(x)|=0$. Nonetheless, $\boldsymbol{\theta}_{f}$ is the zero measure on $\mathbf{X}$, hence $\left\|\boldsymbol{\theta}_{f}\right\|=0$.

For "well behaved" internal functions, the integration of their ${ }^{\circ}$-images with respect to the Loeb measure, as well as of their ${ }^{b}$-images with respect to the pushed down Loeb measure, can be represented by the internal inner product on ${ }^{*} \mathbb{C}^{X}$ :

$$
\langle f, g\rangle=\sum_{x \in X} f(x) g(x) .
$$

3.5. Proposition. Let either $f \in \mathcal{C}(X), g \in \mathcal{M}_{\omega}(X)$, or $f \in \mathcal{C}_{\omega}(X), g \in \mathbb{F}_{1}{ }^{*} \mathbb{C}^{X}$. Then $f g \in \mathcal{M}_{\omega}(X)$, the functions ${ }^{\circ} f: X \rightarrow \mathbb{C}, f^{b}: \mathbf{X} \rightarrow \mathbb{C}$ are integrable with respect to the measures $\lambda_{g}, \boldsymbol{\theta}_{g}$, respectively, and

$$
\mathrm{d} \lambda_{f g}={ }^{\circ} f \mathrm{~d} \lambda_{g}, \quad \mathrm{~d} \boldsymbol{\theta}_{f g}=f^{b} \mathrm{~d} \boldsymbol{\theta}_{g} .
$$

In particular,

$$
\int_{\mathbf{X}} f^{b} \mathrm{~d} \boldsymbol{\theta}_{g}=\int_{X_{\omega}}{ }^{\circ} f \mathrm{~d} \lambda_{g}=\int_{X}{ }^{\circ} f \mathrm{~d} \lambda_{g}={ }^{\circ}\langle f, g\rangle .
$$

Analogous results even hold under weaker assumptions on $f$ and $g$; however, the above formulation is sufficient for our purpose. 


\section{Representing regular Borel measures by internal functions}

Let $\boldsymbol{\mu}: \operatorname{Bo}(\mathbf{X}) \rightarrow \mathbb{C}$ be a fixed regular complex Borel measure on $\mathbf{X}$. In this section we will construct an internal function $f: X \rightarrow{ }^{*} \mathbb{C}$ such that $f \in \mathcal{M}_{\omega}(X), \boldsymbol{\theta}_{f}=\boldsymbol{\mu}$ and $\|f\|_{1} \approx\|\boldsymbol{\mu}\|$.

A finite ordered $(n+1)$-tuple $\beta=\left(B_{0}, B_{1}, \ldots, B_{n}\right)$ of internal subsets of $X$ is called a bounded separated partition of the IMG space $\left(X, E, X_{\omega}\right)$, briefly, a $B S$ partition, if $B_{1}, \ldots, B_{n}$ are nonempty subsets of $X_{\omega}$ with pairwise disjoint observable traces, and $B_{0}=X \backslash\left(B_{1} \cup \ldots \cup B_{n}\right)$. (However, if $X=X_{\omega}$ then the "waste bin" $B_{0}$ may be empty; on the other hand, $B_{0}^{\mathrm{b}} \cap B_{i}^{\mathrm{b}} \neq \emptyset$ may happen for $\left.1 \leq i \leq n\right)$.

For BS partitions $\alpha=\left(A_{0}, A_{1}, \ldots, A_{m}\right), \beta=\left(B_{0}, B_{1}, \ldots, B_{n}\right)$ we put $\alpha \preccurlyeq \beta$ if $A_{0} \subseteq B_{0}$ and for each $1 \leq i \leq m$ there is a $j \leq n$ such that $A_{i} \subseteq B_{j}$. Similarly, $\alpha \wedge \beta=\left(C_{0}, C_{1}, \ldots, C_{k}\right)$, where $C_{0}=A_{0} \cap B_{0}$ and $C_{1}, \ldots, C_{k}$ is the lexicographic enumeration of all the nonempty sets from the list $A_{i} \cap B_{j}, i \leq m, j \leq n,(i, j) \neq$ $(0,0)$. One can readily verify that $\alpha \wedge \beta$ is a BS partition, again,- -in fact the meet of the BS partitions $\alpha, \beta$ with respect to the partial order $\preccurlyeq$.

For any BS partition $\beta=\left(B_{0}, B_{1}, \ldots, B_{n}\right)$ we denote by $\mathcal{F}_{\beta}(\boldsymbol{\mu})$ the set of all internal functions $f: X \rightarrow{ }^{*} \mathbb{C}$ satisfying the following conditions:

$$
\|f\|_{1} \leq\|\boldsymbol{\mu}\|, \quad \text { and } \quad \nu_{f}\left(B_{i}\right)=\boldsymbol{\mu}\left(B_{i}^{b}\right),
$$

for $1 \leq i \leq n$. Obviously, $\mathcal{F}_{\beta}(\boldsymbol{\mu})$ is internal and $\mathcal{F}_{\alpha}(\boldsymbol{\mu}) \subseteq \mathcal{F}_{\beta}(\boldsymbol{\mu})$ for any BS partitions $\alpha \preccurlyeq \beta$.

As the sets $B_{1}^{b}, \ldots, B_{n}^{b} \subseteq \mathbf{X}$ are Borel and pairwise disjoint, we have

$$
\sum_{i=1}^{n}\left|\boldsymbol{\mu}\left(B_{i}^{b}\right)\right| \leq\|f\|_{1} \leq\|\boldsymbol{\mu}\|,
$$

for each $f \in \mathcal{F}_{\beta}(\boldsymbol{\mu})$.

For any $\mathrm{BS}$ partition $\beta=\left(B_{0}, B_{1}, \ldots, B_{n}\right)$ let us introduce the function

$$
f_{\beta}^{\boldsymbol{\mu}}=\sum_{i=1}^{n} \frac{\boldsymbol{\mu}\left(B_{i}^{b}\right)}{\left|B_{i}\right|} \chi_{B_{i}},
$$

where $\chi_{A}: X \rightarrow\{0,1\}$ denotes the characteristic function of the set $A \subseteq X$. Then $f_{\beta}^{\mu}: X \rightarrow{ }^{*} \mathbb{C}$ is an internal function which clearly satisfies the second condition of the definition of the set $\mathcal{F}_{\beta}(\boldsymbol{\mu})$, and also

$$
\left\|f_{\beta}^{\boldsymbol{\mu}}\right\|_{1}=\sum_{i=1}^{n}\left|\boldsymbol{\mu}\left(B_{i}^{b}\right)\right| \leq\|\boldsymbol{\mu}\| .
$$

Hence, $f_{\beta}^{\boldsymbol{\mu}} \in \mathcal{F}_{\beta}(\boldsymbol{\mu})$.

Recall that $\mathcal{B}$ denotes some base of admissible size of the IMG space $\left(X, E, X_{\omega}\right)$. Let $\Pi$ be the set of all BS partitions $\beta=\left(B_{0}, B_{1}, \ldots, B_{n}\right)$ such that $B_{i} \in \mathcal{B}$ for each $i \leq n$. Then $(\Pi, \preccurlyeq)$ clearly is a partially ordered set of admissible size, closed with respect to the meet operation $\alpha \wedge \beta$. Hence, $\left(\mathcal{F}_{\beta}(\boldsymbol{\mu})\right)_{\beta \in \Pi}$ is a downward directed system of admissible size, consisting of nonempty internal sets. By the virtue of saturation,

$$
\mathcal{F}(\boldsymbol{\mu})=\bigcap_{\beta \in \Pi} \mathcal{F}_{\beta}(\boldsymbol{\mu}) \neq \emptyset .
$$

The following technical lemma can be proved by some straightforward computational arguments which can be omitted. 
4.1. Lemma. For each standard $\varepsilon>0$ there is a $\beta=\left(B_{0}, B_{1}, \ldots, B_{n}\right) \in \Pi$ such that

$$
\|\boldsymbol{\mu}\|-\varepsilon<\sum_{i=1}^{n}\left|\boldsymbol{\mu}\left(B_{i}^{b}\right)\right| \leq\|\boldsymbol{\mu}\| .
$$

Then for each $f \in \mathcal{F}_{\beta}(\boldsymbol{\mu})$ we have

$$
\|\boldsymbol{\mu}\|-\varepsilon<\|f\|_{1} \leq\|\boldsymbol{\mu}\|, \quad \text { and } \quad\left|\nu_{f}\right|\left(B_{0}\right)<\varepsilon .
$$

4.2. Proposition. Let $f \in \mathcal{F}(\boldsymbol{\mu})$. Then $f$ satisfies the following conditions:

(a) $f \in \mathcal{M}_{\omega}(X)$;

(b) $\|f\|_{1} \approx\|\boldsymbol{\mu}\| ;$

(c) $\boldsymbol{\theta}_{f}=\boldsymbol{\mu}$.

Proof. (a) We have $\|f\|_{1} \leq\|\boldsymbol{\mu}\|<\infty$. Let $A \subseteq X \backslash X_{\omega}$ be internal. We will prove that $\left|\nu_{f}\right|(A) \approx 0$ by showing $\left|\nu_{f}\right|(A)<\varepsilon$ for each standard $\varepsilon>0$. By lemma 4.1, there is a $\beta=\left(B_{0}, B_{1}, \ldots, B_{n}\right) \in \Pi$ such that $\left|\nu_{g}\right|\left(B_{0}\right)<\varepsilon$ for any $g \in \mathcal{F}_{\beta}(\boldsymbol{\mu})$. As $A_{0} \subseteq B_{0}$ and $f \in \mathcal{F}_{\beta}(\boldsymbol{\mu})$, the conclusion follows.

(b) It suffices to prove $\|\boldsymbol{\mu}\|-\varepsilon<\|f\|_{1}$ for any standard $\varepsilon>0$. By the same lemma, there is a $\beta=\left(B_{0}, B_{1}, \ldots, B_{n}\right) \in \Pi$ such that $\|\boldsymbol{\mu}\|-\varepsilon<\|g\|_{1}$ for any $g \in \mathcal{F}_{\beta}(\boldsymbol{\mu})$. As $f \in \mathcal{F}_{\beta}(\boldsymbol{\mu})$, we are done.

(c) As both the measures $\boldsymbol{\theta}_{f}$ and $\boldsymbol{\mu}$ are regular, it suffices to show that they agree on compact subsets of $\mathbf{X}$, i.e., on sets of the form $A^{b}$ where $A \subseteq X_{\omega}$ is internal. Then

$$
\mathcal{B}_{A}=\left\{B \in \mathcal{B} ; E A \subseteq B \subseteq X_{\omega}\right\}
$$

is a downward directed system of admissible size, and we have

$$
E A=\bigcap \mathcal{B}_{A}, \quad A^{b}=\bigcap_{B \in \mathcal{B}_{A}} B^{b} .
$$

For each $B \in \mathcal{B}_{A}$ the couple $B_{0}=X \backslash B, B_{1}=B$ forms a BS partition $\pi_{B}=$ $\left(B_{0}, B_{1}\right) \in \Pi$. For $g \in \mathcal{F}_{\pi_{B}}(\boldsymbol{\mu})$ we have

$$
\lambda_{g}(B)={ }^{\circ} \nu_{g}(B)=\boldsymbol{\mu}\left(B^{b}\right) .
$$

Thus for $f \in \mathcal{F}(\boldsymbol{\mu}) \subseteq \bigcap_{B \in \mathcal{B}_{A}} \mathcal{F}_{\pi_{B}}(\boldsymbol{\mu})$, using partial $\tau$-smoothness of the Loeb measure $\lambda_{f}$ and outer-open regularity of $\boldsymbol{\mu}$, we obtain

$$
\boldsymbol{\theta}_{f}\left(A^{b}\right)=\lambda_{f}(E A)=\lim _{B \in \mathcal{B}_{A} \downarrow} \lambda_{f}(B)=\lim _{B \in \mathcal{B}_{A} \downarrow} \boldsymbol{\mu}\left(B^{b}\right)=\boldsymbol{\mu}\left(A^{b}\right) .
$$

Propositions 4.2 and 3.1 immediately yield the following consequence.

4.3. Theorem. Let $\boldsymbol{\mu} \in \mathbf{M}(\mathbf{X})$ and $f \in \mathcal{F}(\boldsymbol{\mu})$. Then $\boldsymbol{\mu}=\boldsymbol{\theta}_{f}$, and the observable trace map ${ }^{b}:\left(X_{\omega}, \widetilde{{ }^{*}}\left(X_{\omega}\right), \lambda_{f}\right) \rightarrow(\mathbf{X}, \operatorname{Bo}(\mathbf{X}), \boldsymbol{\mu})$ is surjective, measurable and measure preserving, i.e.,

$$
\mathbf{Y}^{\sharp} \in \widetilde{* \mathcal{P}}\left(X_{\omega}\right), \quad \text { and } \quad \boldsymbol{\mu}(\mathbf{Y})=\lambda_{f}\left(\mathbf{Y}^{\sharp}\right)=\boldsymbol{\theta}_{f}(\mathbf{Y}),
$$

for any $\mathbf{Y} \in \operatorname{Bo}(\mathbf{X})$.

From proposition 4.2 and propositions 3.3 and 3.2, respectively, we also get

4.4. Corollary. $\boldsymbol{\theta}: \mathbb{F}_{1}{ }^{*} \mathbb{C}^{X} \rightarrow \mathbf{M}(\mathbf{X})$ is a surjective $\mathbb{F}^{*} \mathbb{C}$-linear mapping, such that $\left\|\boldsymbol{\theta}_{f}\right\| \leq{ }^{\circ}\|f\|_{1}$ for any $f \in \mathbb{F}_{1}{ }^{*} \mathbb{C}^{X}$. Moreover, for any $\boldsymbol{\mu} \in \mathbf{M}(\mathbf{X})$ there even is an $f \in \mathcal{M}_{\omega}(X)$ such that $\boldsymbol{\mu}=\boldsymbol{\theta}_{f}$ and $\|\boldsymbol{\mu}\|={ }^{\circ}\|f\|_{1}$.

4.5. Corollary. For any function $g \in \mathbb{F}_{1}{ }^{*} \mathbb{C}^{X}$ there is a function $f \in \mathcal{M}_{\omega}(X)$ such that $\boldsymbol{\theta}_{f}=\boldsymbol{\theta}_{g}$ and $\|f\|_{1} \approx\left\|\boldsymbol{\theta}_{g}\right\|$. 


\section{Internal functions inducing zero measures}

In this section we will examine under which conditions the measures $\boldsymbol{\theta}_{f}$ and $\boldsymbol{\theta}_{g}$ coincide, for $f, g \in \mathcal{M}_{\omega}(X)$. To this end it is enough to determine the kernel of the restriction $\boldsymbol{\theta}\left\lceil\mathcal{M}_{\omega}(X)\right.$.

Each measure $\boldsymbol{\mu} \in \mathbf{M}(\mathbf{X})$ is induced by some internal function $f \in \mathcal{M}_{\omega}(X)$ (i.e., $\left.\boldsymbol{\mu}=\boldsymbol{\theta}_{f}\right)$, satisfying $\nu_{f}(X) \approx \boldsymbol{\mu}(\mathbf{X})$ and $\|f\|_{1} \approx\|\boldsymbol{\mu}\|$. On the other hand, from the mere facts $f \in \mathcal{M}_{\omega}(X)$ and $\boldsymbol{\mu}=\boldsymbol{\theta}_{f}$ one can infer just $\|\boldsymbol{\mu}\| \leq{ }^{\circ}\|f\|_{1}$ and not $\|\boldsymbol{\mu}\| \approx\|f\|_{1}$.

In the fairly special case of $E$ coinciding on $X_{\omega}$ with the equality relation we obviously have $\left\|\boldsymbol{\theta}_{f}\right\| \approx\|f\|_{1}$ for any $f \in \mathcal{M}_{\omega}(X)$. However, as shown by example 3.4, this is no more true once $E \cap X_{\omega}^{2} \neq \operatorname{Id}_{X_{\omega}}$. The point is that possible fluctuations of $f$ inside each monad $E\{x\}$ get averaged and compensated passing to the measure $\boldsymbol{\theta}_{f}$; on the other hand, they still contribute to the norm $\|f\|_{1}$, ignoring the equivalence relation $E$, i.e., in fact the topology of $\mathbf{X}$.

Let us denote

$$
\mathcal{M}_{0}(X)=\operatorname{Ker} \boldsymbol{\theta} \cap \mathcal{M}_{\omega}(X)=\left\{f \in \mathcal{M}_{\omega}(X) ; \boldsymbol{\theta}_{f}=0\right\}
$$

the set of all internal functions $f \in \mathcal{M}_{\omega}(X)$ inducing zero measure on $\mathbf{X}$. Obviously, it is an $\mathbb{F}^{*} \mathbb{C}$-linear subspace of ${ }^{*} \mathbb{C}^{X}$.

Using regularity of $\boldsymbol{\theta}_{f}$ and the particular form of compact sets in $\mathbf{X}, \mathcal{M}_{0}(X)$ can be described explicitly, as well. An alternative description can be given using the hyperfinite dimensional representation of the space $\mathbf{C}_{0}(\mathbf{X}) \cong \mathcal{C}_{\omega}(X) / \mathbb{I}_{\infty}{ }^{*} \mathbb{C}^{X}$ from section 1 and (half of) the Riesz representation theorem, namely uniqueness of the measure representing a given bounded linear functional on $\mathbf{C}_{0}(\mathbf{X})$.

5.1. Proposition. For each internal function $g \in \mathcal{M}_{\omega}(X)$ the following conditions are equivalent:

(i) $g \in \mathcal{M}_{0}(X)$, i.e., $\boldsymbol{\theta}_{g}=0$;

(ii) $\lim _{R \in \mathcal{R} \downarrow}{ }^{\circ} \nu_{g}(R A)=0$, for each internal $A \subseteq X_{\omega}$;

(iii) $\langle f, g\rangle \approx 0$, for each $f \in \mathcal{C}_{\omega}(X)$.

Proof. (i) $\Leftrightarrow$ (ii) Due to regularity of $\boldsymbol{\theta}_{g}$, (i) is equivalent to $\boldsymbol{\theta}_{g}(\mathbf{K})=0$ for each compact set $\mathbf{K} \subseteq \mathbf{X}$. This is equivalent to $\lambda_{g}(E A)=0$ for each internal set $A \subseteq X_{\omega}$, and - as

$$
\lambda_{g}(E A)=\lim _{R \in \mathcal{R} \downarrow} \lambda_{g}(R A)=\lim _{R \in \mathcal{R} \downarrow}{ }^{\circ} \nu_{g}(R A)
$$

by partial $\tau$-smoothness of $\lambda_{g}$-to (ii), as well.

(i) $\Leftrightarrow$ (iii) follows from the uniqueness part of the Riesz representation theorem through the equality

$$
\int f^{b} \mathrm{~d} \boldsymbol{\theta}_{g}={ }^{\circ}\langle f, g\rangle
$$

for $f \in \mathcal{C}_{\omega}(X), g \in \mathcal{M}_{\omega}(X)$,- see proposition 3.5.

Similarly as in section 1 , there is a chain of $\mathbb{F}^{*} \mathbb{C}$-linear subspaces of the hyperfinite dimensional vector space ${ }^{*} \mathbb{C}^{X}$ :

$$
\mathbb{I}_{1}{ }^{*} \mathbb{C}^{X} \subseteq \mathcal{M}_{0}(X) \subseteq \mathcal{M}_{\omega}(X) \subseteq \mathbb{F}_{1}^{*} \mathbb{C}^{X}
$$


As shown in example 3.4, the inclusion $\mathbb{I}_{1} * \mathbb{C}^{X} \subseteq \mathcal{M}_{0}(X)$ is proper, in general. It can easily be seen that both $\mathcal{M}_{0}(X), \mathcal{M}_{\omega}(X)$ are closed in ${ }^{*} \mathbb{C}^{X}$ with respect to the pseudonorm ${ }^{\circ}\|\cdot\|_{1}$, and so are the quotients $\mathcal{M}_{0}(X) / \mathbb{I}_{1}{ }^{*} \mathbb{C}^{X}, \mathcal{M}_{\omega}(X) / \mathbb{I}_{1}{ }^{*} \mathbb{C}^{X}$ in the nonstandard hull $\mathbb{F}_{1}{ }^{*} \mathbb{C}^{X} / \mathbb{I}_{1}{ }^{*} \mathbb{C}^{X}$ of the hyperfinite dimensional Banach space $\left(* \mathbb{C}^{X},\|\cdot\|_{1}\right)$.

For each $g \in \mathcal{M}_{\omega}(X)$ let us denote by

$$
[g]=g+\mathcal{M}_{0}(X)
$$

its equivalence class in the quotient $\mathcal{M}_{\omega}(X) / \mathcal{M}_{0}(X)$. Then the formula

$$
\|[g]\|=\inf _{h \in[g]}{ }^{\circ}\|h\|_{1}=\inf \left\{{ }^{\circ}\|h\|_{1} ; g-h \in \mathcal{M}_{0}(X)\right\}
$$

defines a norm on the vector space $\mathcal{M}_{\omega}(X) / \mathcal{M}_{0}(X)$, turning it into a (classical) Banach space (over $\mathbb{C}$ ).

For each $g \in \mathcal{M}_{\omega}(X)$ we have $\left\|\boldsymbol{\theta}_{g}\right\| \leq{ }^{\circ}\|g\|_{1}$. On the other hand, by corollary 4.5,

$$
\left(\forall g \in \mathcal{M}_{\omega}(X)\right)\left(\exists h \in \mathcal{M}_{\omega}(X)\right)\left(\boldsymbol{\theta}_{g}=\boldsymbol{\theta}_{h} \&\left\|\boldsymbol{\theta}_{g}\right\|_{1}={ }^{\circ}\|h\|_{1}\right) .
$$

Then

$$
\|[g]\|={ }^{\circ}\|h\|_{1}=\left\|\boldsymbol{\theta}_{g}\right\|
$$

Summing up we get

5.2. Theorem. Let $\left(X, E, X_{\omega}\right)$ be a hyperfinite condensating IMG space and $\mathbf{X} \cong X^{b}$. Then the mapping $[g] \mapsto \boldsymbol{\theta}_{g}$ is an isometric linear isomorphism of the Banach space $\mathcal{M}_{\omega}(X) / \mathcal{M}_{0}(X)$ onto the Banach space $\mathbf{M}(\mathbf{X})$.

However, unless $\mathcal{M}_{0}(X)=\mathbb{I}_{1}{ }^{*} \mathbb{C}^{X}$, this gives just a representation of $\mathbf{M}(\mathbf{X})$ as a subspace of the quotient

$$
\mathcal{M}_{\omega}(X) / \mathcal{M}_{0}(X) \cong \frac{\mathcal{M}_{\omega}(X) / \mathbb{I}_{1} * \mathbb{C}^{X}}{\mathcal{M}_{0}(X) / \mathbb{I}_{1} * \mathbb{C}^{X}}
$$

of subspaces of $\mathbb{F}_{1} * \mathbb{C}^{X} / \mathbb{I}_{1} * \mathbb{C}^{X}$, but not as a subspace of this nonstandard hull itself.

\section{The algebra of measures on a group}

An IMG group is a triple $\left(G, G_{0}, G_{\omega}\right)$ consisting of an internal group $G$, and two its subgroups $G_{0} \subseteq G_{\omega} \subseteq G$, such that $G_{0}$ is monadic, $G_{\omega}$ is galactic, and $G_{0}$ is normal in $G_{\omega}$. Similarly like in section 1 , the monad $G_{0}$ is viewed as the subgroup of infinitesimals in $G$, and so is the galaxy $G_{\omega}$ as the subgroup of its elements situated in front of some horizon.

With any IMG group, in fact, two IMG spaces can be associated: $\left(G, E_{l}, G_{\omega}\right)$ and $\left(G, E_{r}, G_{\omega}\right)$, where $E_{l}, E_{r}$ is the left and the right equivalence relation on $G$ corresponding to $G_{0}$, respectively. Though they agree on the galaxy $G_{\omega}$, they still may induce different uniformities on $G$ and even on $G_{\omega}$. However, as far as we are interested just in the topology of the galaxy $G_{\omega}$ and its observable trace $G^{b}$, we need not care about this subtle difference.

The groups $G, G_{\omega}$, as well as the observable trace $G^{b}=G_{\omega} / G_{0}$, endowed with the topologies described in section 1 , become topological groups, and the observable 
trace map $x \mapsto x^{b}$ is a continuous surjective homomorphism of topological groups $G_{\omega} \rightarrow G^{b}$.

The systems $\mathcal{R}$ and $\mathcal{V}$ can be supplied by a single system $\mathcal{S}$ of admissible size, directed both upward and downward, consisting of symmetric internal subsets of $G$, such that

$$
G_{0}=\bigcap \mathcal{S}, \quad G_{\omega}=\bigcup \mathcal{S},
$$

and

$$
\begin{gathered}
(\forall S \in \mathcal{S})(\exists R, T \in \mathcal{S})(R \cdot R \subseteq S \quad \& \quad S \cdot S \subseteq T), \\
(\forall S, T \in \mathcal{S})(\exists R \in \mathcal{S})(\forall x \in T)\left(x R x^{-1} \subseteq S\right) .
\end{gathered}
$$

Again, an IMG group $\left(G, G_{0}, G_{\omega}\right)$ is called condensating if it is condensating as an IMG space (no matter whether $\left(G, E_{l}, G_{\omega}\right)$ or $\left(G, E_{r}, G_{\omega}\right)$ ). This is equivalent to relative compactness of all the sets $S \in \mathcal{S}$. The observable trace $G^{b}$ of a condensating IMG group $\left(G, G_{0}, G_{\omega}\right)$ is a locally compact topological group.

The other way round, every Hausdorff locally compact group $\mathbf{G}$ is isomorphic to the observable trace $G^{b}$ of some condensating IMG group $\left(G, G_{0}, G_{\omega}\right)$. In particular, this works with $G={ }^{*} \mathbf{G}, G_{0}=\operatorname{mon}(1)$ and $G_{\omega}=\operatorname{ns}\left({ }^{*} \mathbf{G}\right)$, cf. [25] and [24].

However, in comparison with topological spaces (cf. section 1), the situation becomes essentially different if one additionally requires $G$ to be hyperfinite. As proved by Gordon [8], every Abelian metrizable, locally compact and $\sigma$-compact group can be represented as $\mathbf{G} \cong G^{b}$ with $G$ hyperfinite Abelian. Since the Haar measure on G can be obtained by pushing down the Loeb measure induced by the constant function $x \mapsto 1 /|S|$ on $G$, for any $S \in \mathcal{S}$, it can easily be seen that every $\mathbf{G}$ admitting such a representation is necessarily unimodular. On the other hand, as shown in [2], the compact (hence unimodular) metrizable group $\mathrm{SO}(3)$ cannot be represented as $G^{b}$ with hyperfinite $G$. A reasonable intrinsic characterization of locally compact groups $\mathbf{G}$, isomorphic to observable traces $G^{b}$ of hyperfinite condensating IMG groups $\left(G, G_{0}, G_{\omega}\right)$, is still missing.

In view of these facts, the results of the present section should be understood in the following way: Whenever a classical locally compact group $\mathbf{G}$ is isomorphic (as a topological group) to the observable trace $G^{b}=G_{\omega} / G_{0}$ of a hyperfinite condensating IMG group $\left(G, G_{0}, G_{\omega}\right)$, then the algebra $\mathbf{M}(\mathbf{G})$ of regular complex Borel measures on $\mathbf{G}$ is isomorphic to the algebra $\mathcal{M}_{\omega}(G) / \mathcal{M}_{0}(G)$ described below.

So let $\left(G, G_{0}, G_{\omega}\right)$ be a fixed hyperfinite condensating IMG group, and $\mathbf{G}=G^{b}$ be its observable trace. Then the triple $\left(G^{2}, G_{0}^{2}, G_{\omega}^{2}\right)$ obviously satisfies analogous conditions, hence it is a hyperfinite condensating IMG group, as well. The corresponding observable trace map $G_{\omega}^{2} \rightarrow \mathbf{G}^{2}$, given by

$$
(x, y) \mapsto(x, y)^{b}=\left(x^{b}, y^{b}\right)
$$

induces a canonic isomorphism of topological groups $\left(G^{2}\right)^{b}=G_{\omega}^{2} / G_{0}^{2}$ and $\mathbf{G}^{2}=$ $\left(G^{b}\right)^{2}=\left(G_{\omega} / G_{0}\right)^{2}$, justifying their identification. Then

$$
\mathbf{Z}^{\sharp}=\left\{(x, y) \in G_{\omega}^{2} ;\left(x^{b}, y^{b}\right) \in \mathbf{Z}\right\}
$$

denotes the pretrace of any set $\mathbf{Z} \subseteq \mathbf{G}^{2}$. 
For any $Y \subseteq G$ we put

$$
Y^{(2)}=\left\{(s, t) \in G^{2} ; s t \in Y\right\}
$$

and similarly for subsets of G. Obviously, $Y^{(2)}$ is internal (in $\widetilde{*}(G)$ ) for internal (Borel) $Y \subseteq G$, and $\mathbf{Y}^{(2)}$ is Borel in $\mathbf{G}^{2}$ for any Borel $\mathbf{Y} \subseteq \mathbf{G}$. Then we trivially have

$$
\left(\mathbf{Y}^{(2)}\right)^{\sharp}=\left(\mathbf{Y}^{\sharp}\right)^{(2)} \cap G_{\omega}^{2},
$$

for any $\mathbf{Y} \subseteq \mathbf{G}$.

For internal functions $f, g: G \rightarrow{ }^{*} \mathbb{C}^{G}$ their "tensor product" $f \otimes g: G^{2} \rightarrow{ }^{*} \mathbb{C}$ and convolution $f * g: G \rightarrow{ }^{*} \mathbb{C}$ are defined in the usual way:

$$
\begin{gathered}
(f \otimes g)(x, y)=f(x) g(y), \\
(f * g)(x)=\sum_{s t=x} f(s) g(t)=\sum_{t \in G} f\left(x t^{-1}\right) g(t),
\end{gathered}
$$

for $x, y \in G$. Obviously, both $f \otimes g$ and $f * g$ are internal. Then, for each internal set $A \subseteq G$, we have

$$
\nu_{f * g}(A)=\sum_{x \in A}(f * g)(x)=\sum_{s t \in A} f(s) g(t)=\nu_{f \otimes g}\left(A^{(2)}\right)
$$

Similarly, the inequality

$$
\|f * g\|_{1} \leq\|f\|_{1}\|g\|_{1}
$$

can be verified by a straightforward computation. It follows that, with multiplication given by the convolution, $\mathbb{F}_{1}{ }^{*} \mathbb{C}^{G}$ is an $\mathbb{F}^{*} \mathbb{C}$-subalgebra of the internal Banach

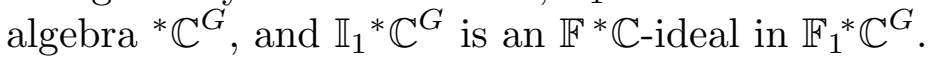

We also denote

$$
{ }_{t} f(x)=f(t x), \quad f_{t}(x)=f(x t),
$$

the left and the right shift, respectively, of a function $f \in{ }^{*} \mathbb{C}^{X}$ by an element $t \in G$.

Next we will show that both the subspaces $\mathcal{M}_{0}(G), \mathcal{M}_{\omega}(G)$ of $* \mathbb{C}^{G}$ are closed with respect to shifts by elements $t \in G_{\omega}$, and $\mathcal{M}_{\omega}(G)$ is closed with respect to convolution, i.e., it is an $\mathbb{F}^{*} \mathbb{C}$-subalgebra of the internal Banach algebra $* \mathbb{C}^{G}$.

6.1. Proposition. (a) If $f \in \mathcal{M}_{\omega}(G)$ and $t \in G_{\omega}$, then ${ }_{t} f, f_{t}, \in \mathcal{M}_{\omega}(G)$.

(b) If $f \in \mathcal{M}_{0}(G)$ and $t \in G_{\omega}$, then ${ }_{t} f, f_{t}, \in \mathcal{M}_{0}(G)$.

(c) If $f, g \in \mathcal{M}_{\omega}(G)$, then $f * g \in \mathcal{M}_{\omega}(G)$.

Proof. We will consider just the right shifts, both in (a) and (b).

(a) Clearly, $\left\|f_{t}\right\|_{1}=\|f\|_{1}$ for any $f \in{ }^{*} \mathbb{C}^{G}, t \in G$. Thus it suffices to verify $\left|\nu_{f_{t}}\right|(A) \approx 0$, for internal $A \subseteq G \backslash G_{\omega}$ and $t \in G_{\omega}$. Then $A t \subseteq G \backslash G_{\omega}$ is internal, as well, and, obviously,

$$
\left|\nu_{f_{t}}\right|(A)=\left|\nu_{f}\right|(A t) \approx 0 .
$$

(b) In view of (a) and proposition 5.1, we have just to show that $\left\langle h, f_{t}\right\rangle \approx 0$, whenever $h \in \mathcal{C}_{\omega}(G), f \in \mathcal{M}_{0}(G)$ and $t \in G_{\omega}$. Then, obviously, $h_{t^{-1}} \in \mathcal{C}_{\omega}(G)$, hence

$$
\left\langle h, f_{t}\right\rangle=\left\langle h_{t^{-1}}, f\right\rangle \approx 0
$$


(c) Assume that $\|f\|_{1},\|g\|_{1}<\infty$ and both $\left|\nu_{f}\right|(A),\left|\nu_{g}\right|(A)$ are infinitesimal, for any internal $A \subseteq G \backslash G_{\omega}$. It remains to show that, in such a case, $\left|\nu_{f * g}\right|(A)$ is infinitesimal, as well. One immediately gets

$$
\left|\nu_{f * g}\right|(A)=\sum_{x \in A}|(f * g)(x)|=\sum_{x \in A}\left|\sum_{t \in G} f\left(x t^{-1}\right) g(t)\right| \leq \sum_{t \in G} h(t)|g(t)|,
$$

where

$$
h(t)=\sum_{x \in A}\left|f\left(x t^{-1}\right)\right|=\sum_{x \in A}\left|f_{t^{-1}}(x)\right|
$$

Obviously, $h \in{ }^{*} \mathbb{C}^{G}$, and $0 \leq h(t) \leq\|f\|_{1}$ for any $t \in G$. Let us denote

$$
T=\{t \in G ; h(t) \approx 0\}
$$

Then $T$ is a monadic, $G_{\omega}$ is galactic, and $G_{\omega} \subseteq T$ by (b). Thus there is a hyperfinite set $B$, such that $G_{\omega} \subseteq B \subseteq T$. Now, we have $G \backslash B \subseteq G \backslash G_{\omega}$, and

$$
\begin{aligned}
\left|\nu_{f * g}\right|(A) & \leq \sum_{t \in B} h(t)|g(t)|+\sum_{t \in G \backslash B} h(t)|g(t)| \\
& \leq\|g\|_{1} \max _{t \in B} h(t)+\|f\|_{1}\left|\nu_{g}\right|(G \backslash B) \approx 0 .
\end{aligned}
$$

The product of Loeb measures $\lambda_{f}, \lambda_{g}$ is defined in the usual way. However, it should be noted that not even all internal subsets of $G_{\omega}$ are necessarily measurable with respect to $\lambda_{f} \times \lambda_{g}$. Fortunately, the following fact is still true - see [4] or [1].

6.2. Lemma. Let $f, g \in \mathbb{F}_{1}{ }^{*} \mathbb{C}^{G}$ and $Z \subseteq G^{2}$ be a set measurable with respect to the product measure $\lambda_{f} \times \lambda_{g}$. Then $Z$ is measurable with respect to the Loeb measure $\lambda_{f \otimes g}$, as well, and

$$
\left(\lambda_{f} \times \lambda_{g}\right)(Z)=\lambda_{f \otimes g}(Z)
$$

Recall that the Banach space of regular complex Borel measures on $\mathbf{G}$ is in fact a Banach algebra, once multiplication of measures is defined as convolution

$$
\left(\boldsymbol{\mu}_{1} * \boldsymbol{\mu}_{2}\right)(\mathbf{B})=\left(\boldsymbol{\mu}_{1} \times \boldsymbol{\mu}_{2}\right)\left(\mathbf{B}^{(2)}\right)
$$

for $\boldsymbol{\mu}_{1}, \boldsymbol{\mu}_{2} \in \mathbf{M}(\mathbf{G}), \mathbf{B} \in \mathrm{Bo}(\mathbf{G})$, see, e.g., [16].

As the the set-theoretical operations of complement and arbitrary unions are preserved by the preimage under the observable trace map $G_{\omega}^{2} \rightarrow \mathbf{G}^{2}$, we obviously have

6.3. Lemma. Let $f, g \in \mathbb{F}_{1}{ }^{*} \mathbb{C}^{G}$ and $\mathbf{Z} \subseteq \mathbf{G}^{2}$ be measurable with respect to the product measure $\boldsymbol{\theta}_{f} \times \boldsymbol{\theta}_{g}$. Then $\mathbf{Z}^{\sharp} \subseteq G_{\omega}^{2}$ is measurable with respect to the product of the Loeb measures $\lambda_{f}, \lambda_{g}$ and

$$
\left(\lambda_{f} \times \lambda_{g}\right)\left(\mathbf{Z}^{\sharp}\right)=\left(\boldsymbol{\theta}_{f} \times \boldsymbol{\theta}_{g}\right)(\mathbf{Z}) .
$$


6.4. Proposition. Let $f, g \in \mathcal{M}_{\omega}(G)$. Then $\boldsymbol{\theta}_{f * g}=\boldsymbol{\theta}_{f} * \boldsymbol{\theta}_{g}$.

Proof. As the measures on both sides are regular, it is enough to show that they coincide on compact subsets of $\mathbf{G}$. If $\mathbf{K} \subseteq \mathbf{G}$ is compact, then $\mathbf{K}=A^{\text {b }}$ for some internal $A \subseteq G_{\omega}$. Hence $\mathbf{K}^{\sharp}=G_{0} A=\bigcap_{S \in \mathcal{S}} S A$ and $\left(\mathbf{K}^{(2)}\right)^{\sharp}=\left(G_{0} A\right)^{(2)} \cap G_{\omega}^{2}$. Therefore,

$$
\begin{aligned}
\left(\boldsymbol{\theta}_{f} * \boldsymbol{\theta}_{g}\right)(\mathbf{K}) & =\left(\boldsymbol{\theta}_{f} \times \boldsymbol{\theta}_{g}\right)\left(\mathbf{K}^{(2)}\right)=\left(\lambda_{f} \times \lambda_{g}\right)\left(\left(G_{0} A\right)^{(2)} \cap G_{\omega}^{2}\right) \\
& =\left(\lambda_{f} \times \lambda_{g}\right)\left(\left(G_{0} A\right)^{(2)}\right),
\end{aligned}
$$

by the virtue of lemma 6.3 and the fact that both $\left|\lambda_{f}\right|,\left|\lambda_{g}\right|$ are finite and concentrated on $G_{\omega}$, - see proposition 3.2. Thus the monadic set $\left(G_{0} A\right)^{(2)} \subseteq G^{2}$ is measurable with respect to the product of the Loeb measures $\lambda_{f}, \lambda_{g}$. By lemma 6.2 and partial $\tau$-smoothness of Loeb measures,

$$
\begin{aligned}
\left(\lambda_{f} \times \lambda_{g}\right)\left(\left(G_{0} A\right)^{(2)}\right) & =\lambda_{f \otimes g}\left(\left(G_{0} A\right)^{(2)}\right)=\lim _{S \in \mathcal{S} \downarrow} \lambda_{f \otimes g}\left((S A)^{(2)}\right) \\
& =\lim _{S \in \mathcal{S} \downarrow}{ }^{\circ} \nu_{f \otimes g}\left((S A)^{(2)}\right)=\lim _{S \in \mathcal{S} \downarrow}{ }^{\circ} \nu_{f * g}(S A) \\
& =\lim _{S \in \mathcal{S} \downarrow} \lambda_{f * g}(S A)=\lambda_{f * g}\left(G_{0} A\right)=\boldsymbol{\theta}_{f * g}(\mathbf{K}) .
\end{aligned}
$$

6.5. Corollary. $\mathcal{M}_{0}(G)=\operatorname{Ker} \boldsymbol{\theta} \cap \mathcal{M}_{\omega}(G)$ is an $\mathbb{F}^{*} \mathbb{C}$-ideal in the $\mathbb{F}^{*} \mathbb{C}$-subalgebra $\mathcal{M}_{\omega}(G)$ of the hyperfinite dimensional internal Banach algebra $\left({ }^{*} \mathbb{C}^{G}, *,\|\cdot\|_{1}\right)$.

Thus the quotient $\mathcal{M}_{\omega}(G) / \mathcal{M}_{0}(G)$ naturally becomes a (classical) Banach algebra (over $\mathbb{C}$ ). From proposition 6.4 and theorem 5.2 we get our final result.

6.6. Theorem. Let $\left(G, G_{0}, G_{\omega}\right)$ be a hyperfinite condensating IMG group, and $\mathbf{G} \cong G^{b}$. Then the mapping $[g] \mapsto \boldsymbol{\theta}_{g}$ is an isometric isomorphism of the Banach algebra $\mathcal{M}_{\omega}(G) / \mathcal{M}_{0}(G)$ onto the Banach algebra $\mathbf{M}(\mathbf{G})$.

\section{REFERENCES}

[1] S. Albeverio, J. E. Fenstad, R. Høegh-Krohn and T. Lindstrøm, Nonstandard Methods in Stochastic Analysis and Mathematical Physics, Academic Press, London-New York-Orlando, 1986.

[2] M. A. Alekseev, L. Yu. Glebskii and E. I. Gordon, On approximations of groups, group actions and Hopf algebras, Representation Theory, Dynamical Systems, Combinatorial and Algebraic Methods III (A. M. Vershik, ed.), vol. 256, Russian Academy of Sciences, St. Petersburg Branch of V. A. Steklov's Institute, Zapiski nauchnykh seminarov POMI, 1999, pp. 224-262 (Russian); English transl., J. Math. Sciences 107 (2001), 4305-4332.

[3] J. M. Aldaz, A characterization of universal Loeb measurability for completely regular Hausdorff spaces, Can. J. Math. 44 (1992), 673-690.

[4] R. M. Anderson, A nonstandard representation for Brownian motion and Itô integration, Israel J. Math. 25 (1976), 15-46.

[5] R. M. Anderson, Star-finite representations of measure spaces, Trans. Amer. Math. Soc. 271 (1982), 667-687.

[6] N. Cutland, Nonstandard measure theory and its applications, Bull. London Math. Soc. 15 (1983), 529-589.

[7] L. Yu. Glebsky and E. I. Gordon, On approximations of topological groups by finite algebraic systems, preprint on http://arxiv.org/list.math.FA/0201, math.GR-0201101 v1 11 Jan 2002.

[8] E. I. Gordon, Nonstandard Methods in Commutative Harmonic Analysis, Translations of mathematical monographs vol. 164, Amer. Math. Soc., Providence, R. I., 1997. 
[9] E. I. Gordon, On approximation of topological groups and their representations, Doklady RAN 345 (1995), 593-595. (Russian)

[10] E. I. Gordon and I. A. Korchagina, Hyperfinite approximations of compact groups and their representations, Siberian Math. J. 36 (1995), 308-327. (Russian)

[11] E. I. Gordon and A. M. Vershik, Groups that are locally embeddable in the class of finite groups, St. Peterburg Math. J. 9 (1998), 49-67.

[12] J. Guričan and P. Zlatoš, Biequivalences and topology in the alternative set theory, Comment. Math. Univ. Carolinae 26 (1985), 525-552.

[13] C. W. Henson, On the nonstandard representation of measures, Trans. Amer. Math. Soc. 172 (1972), 437-446.

[14] C. W. Henson, Analytic sets, Baire sets and the standard part map, Can. J. Math. 31 (1979), 663-672.

[15] C. W. Henson and L. C. Moore, Nonstandard analysis and the theory of Banach spaces, Nonstandard Analysis, Recent Developments (A. E. Hurd, ed.), Springer-Verlag, BerlinHeidelberg-New York, 1983, pp. 27-112.

[16] E. Hewitt and K. A. Ross, Abstract Harmonic Analysis I, Springer-Verlag, Berlin-GöttingenHeidelberg, 1963.

[17] D. Landers and L. Rogge, Universal Loeb measurability of sets and of the standard part map with applications, Trans. Amer. Math. Soc. 304 (1987), 229-243.

[18] P. A. Loeb, A nonstandard representation of measurable spaces $L_{\infty}$ and $L_{\infty}^{*}$, Contributions to Nonstandard Analysis (W. A. J. Luxemburg, A. Robinson, eds.), North-Holland, Amsterdam, 1972, pp. 65-80.

[19] P. A. Loeb, A nonstandard representation of Borel measures and $\sigma$-finite measures, Victoria Symposium on Nonstandard Analysis (A. E. Hurd, P. A. Loeb, eds.), LNM, vol. 369, SpringerVerlag, Berlin-Heidelberg-New York, 1974, pp. 144-152.

[20] P. A. Loeb, Conversion from nonstandard to standard measure spaces and applications to probability theory, Trans. Amer. Math. Soc. 211 (1975), 113-122.

[21] P. A. Loeb, Weak limits of measures and the standard part map, Proc. Amer. Math. Soc. 77 (1979), 128-135.

[22] J. Mlček and P. Zlatoš, The Ramsey structure of A-determined sets in a $\kappa$-saturated universe, Proc. Logic Colloquium, Prague '98, Lecture Notes in Logic (S. R. Buss, P. Hájek, P. Pudlák, eds.), vol. 13, Association for Symbolic Logic, A. K. Peters, Nattick, Mass., 1998, pp. 316-333.

[23] J. Mlček and P. Zlatoš, Some Ramsey type theorems for countably determined sets, Arch. Math. Logic 41 (2002), 619-630.

[24] R. Parikh, A nonstandard theory of topological groups, Applications of Model Theory to Algebra, Analysis and Probability (W. A. J. Luxemburg, ed.), Proceedings of an International Symposium on Non-Standard Analysis, Holt, Rinehart and Winston, New York etc., 1969, pp. 279-284.

[25] A. Robinson, Non-Standard Analysis, North-Holland, Amsterdam, 1966.

[26] D. Ross, Loeb measures and probability, Nonstandard Analysis, Theory and Applications (L. O. Arkeryd, N. J. Cutland and C. W. Henson, eds.), NATO ASI Series C, Mathematical and Physical Sciences, vol. 493, Kluwer Academic Publishers, Dordrecht-Boston-London, 1997, pp. 91-120.

[27] W. Rudin, Real and Complex Analysis, McGraw-Hill, New York, 1974.

[28] K. D. Stroyan and J. M. Bayod, Foundations of Infinitesimal Stochastic Analysis, NorthHolland, Amsterdam, 1986.

[29] P. Vopěnka, Mathematics in the Alternative Set Theory, Teubner-Verlag, Leipzig, 1979.

[30] P. Vopěnka, Introduction to Mathematics in the Alternative Set Theory, Izdatel'stvo Instituta matematiki, Novosibirsk, 2004. (Russian)

[31] P. Zlatoš, Indiscernibility and accessibility, Appendix in [30]. (Russian)

Faculty of Mathematics, Physics and Informatics, Comenius University, Mlynská Dolina, 84248 Bratislava, Slovakia

E-mail addresses: ziman@fmph.uniba.sk, zlatos@fmph.uniba.sk 